Sādhanā Vol. 27, Part 3, June 2002, pp. 408-409. () Printed in India

\title{
Comments on the criticism of Sarvajit Singh on the paper 'Surface waves in fibre-reinforced anisotropic elastic media'
}

\author{
P R SENGUPTA and SISIR NATH \\ Department of Mathematics, University of Kalyani, Kalyani 741235, India
}

MS received 24 April 2002

\begin{abstract}
Though the decoupling of displacement potentials in case of fibrereinfoced anisotropic elastic media is not possible in the general case, an attempt has been made to decouple the equation as a particular case. The assumptions made in this paper satisfy both set of equations formed by the displacement potentials, which is the main feature of the criticism made by Sarvajit Singh.
\end{abstract}

Keywords. Decoupling; displacement potentials; fibre-reinforced medium; utopian.

\section{Introduction}

Recently, constructive criticism has been made by Sarvajit Singh (this issue, pp 405-407), henceforth referred to simply as SS, regarding a research paper entitled 'Surface waves in fibre-reinforced anisotropic elastic media' by Sengupta \& Nath (2001). In case of anisotropic media potential solutions have been presented in the standard book of Green \& Zerna (1963). Though the decoupling of displacement potentials in case of fibre-reinforced anisotropic elastic media is not possible in the general case, an attempt has been made to decouple the equation as a particular case. The assumptions made in this paper satisfy both set of equations formed by the displacement potentials. It is true that the consideration of the particular case has not been mentioned in the paper by Sengupta \& Nath (2001).

\section{Discussions}

Equations (1) and (2), as mentioned by SS, are inadvertent errors. Equations (3) and (4) are correct as set out by SS. Now if we consider in (3) and (4) of SS the following assumptions

$$
\alpha+2 \mu_{L}=2 \mu_{T},
$$

and that the interactions of $\beta$ in the neighbourhood of any point tend to zero, i.e. $\beta \rightarrow 0$, then

$$
\begin{aligned}
& \lambda+2 \alpha+4 \mu_{L}-2 \mu_{T}+\beta=\lambda+\alpha+2 \mu_{L}[=p, \text { say, in the limit }], \\
& \lambda+\alpha+2 \mu_{L}=\lambda+2 \mu_{T}[=q, \text { say }], \\
& \alpha+3 \mu_{L}-2 \mu_{T}+\beta=\mu_{L}[=r, \text { say }], \\
& \mu_{L}=2 \mu_{T}-\mu_{L}-\alpha[=s, \text { say }],
\end{aligned}
$$


and (3) and (4) of SS reduce to the following form in the limit as

$$
\begin{gathered}
\frac{\partial}{\partial X_{1}}\left[p \frac{\partial^{2} \phi}{\partial X_{1}^{2}}+q \frac{\partial^{2} \varphi}{\partial X_{2}^{2}}-\rho \frac{\partial^{2} \varphi}{\partial t^{2}}\right]-\frac{\partial}{\partial X_{2}}\left[r \frac{\partial^{2} \psi}{\partial X_{1}^{2}}+s \frac{\partial^{2} \psi}{\partial X_{2}^{2}}-\rho \frac{\partial^{2} \psi}{\partial t^{2}}\right]=0, \\
\frac{\partial}{\partial X_{2}}\left[p \frac{\partial^{2} \varphi}{\partial X_{1}^{2}}+q \frac{\partial^{2} \varphi}{\partial X_{2}^{2}}-\rho \frac{\partial^{2} \varphi}{\partial t^{2}}\right]-\frac{\partial}{\partial X_{1}}\left[r \frac{\partial^{2} \psi}{\partial X_{1}^{2}}+s \frac{\partial^{2} \psi}{\partial X_{2}^{2}}-\rho \frac{\partial^{2} \psi}{\partial t^{2}}\right]=0,
\end{gathered}
$$

which are identically and simultaneously satisfied if

$$
\begin{aligned}
& {\left[p \frac{\partial^{2} \varphi}{\partial x_{1}^{2}}+q \frac{\partial^{2} \varphi}{\partial x_{2}^{2}}-\rho \frac{\partial^{2} \varphi}{\partial t^{2}}\right]=0,} \\
& {\left[r \frac{\partial^{2} \psi}{\partial x_{1}^{2}}+s \frac{\partial^{2} \psi}{\partial x_{2}^{2}}-\rho \frac{\partial^{2} \psi}{\partial t^{2}}\right]=0,}
\end{aligned}
$$

which are the equations considered by Sengupta \& Nath (2001) as (10) and (11) in their paper. Now

$$
\alpha+2 \mu_{L}=2 \mu_{T}
$$

may be possible for different concrete and steel structures. It is mentioned that Chattopadhyay \& Venkateswarlu (1998) considered

$$
\alpha=-1.28 \quad 10^{9} \mathrm{Nm}^{-2}, \quad \mu_{L}=5.6610^{9} \mathrm{Nm}^{-2}, \quad \mu_{T}=2.4610^{9} \mathrm{Nm}^{-2} .
$$

Though $\beta \rightarrow 0$, might seem utopian in its form, there are examples of this type of assumption for a particular case, as seen frequently in different branches of science, viz. the consideration of the inertia term $\rho \ddot{v}=0$ in thermo-elasticity (Nowacki 1976), the consideration of $\beta=\infty$, where $\beta$ denotes the conduction of electricity, in magneto-elasticity (Nowacki 1976) etc. In thermo-elasticity, the isothermal and adiabatic changes in heat conditions are not possible in an elastic medium in practice. Couple-stress theory of elasticity, micropolar theory of elasticity, void theory of elasticity are also considered utopian theories by some research workers but developments in these branches from ideal to real are still going on.

\section{References}

Chattopadhyay A, Venkateswarlu R L K 1998 stresses produced in a fibre-reinforced half space due to a moving load. Bull. Calutta.Math.Soc 90: 337-342

Green. A E, Zerna W 1963 Theoretical elasticity (London: Oxford University Press) pp 180-183

Nowacki W 1976 Trends in applications of pure mathematics to mechanics (ed.) Gaetano Fichera (Pitman) pp 267,276

Sengupta P R, Nath S 2001 Surface waves in fibre-reinforced anisotropic elastic media. Sādhanā 26: $363-370$ 